\title{
TRADUCCIÓN, ADAPTACIÓN AL ESPAÑOL Y VALIDACIÓN DE LA ESCALA DE BIENESTAR MENTAL DE WARWICK-EDINBURGH EN UNA MUESTRA DE ADULTOS MAYORES ARGENTINOS
}

\author{
Daniel Serrani Azcurra* \\ Facultad de Psicología Universidad Nacional de Rosario - Argentina
}

Recibido, febrero 16/2014

Concepto de evaluación, octubre 21/2014

Aceptado, noviembre 28/2014
Referencia: Serrani Azcurra, D. (2015). Traducción, adaptación al español y validación de la escala de bienestar mental de Warwick-Edinburgh en una muestra de adultos mayores argentinos. Acta Colombiana de Psicología, 18(1), 79-93. DOI: 10.14718/ACP.2015.18.1.8

\begin{abstract}
Resumen
El estudio analiza las propiedades psicométricas y la estructura factorial de la Escala de Bienestar Mental de WarwickEdinburgh luego de realizar la traducción directa y revertida y su adaptación al español. Se validó la escala mediante su aplicación a una muestra de 910 adultos mayores argentinos, con edades comprendidas entre 71 y 96 años (media 81.4 años; DE 10.9 años). Se obtuvieron los siguientes indicadores: distribución de respuestas, consistencia interna, confiabilidad test-retest, validez de constructo, y se realizó un análisis factorial exploratorio y confirmatorio. En cuatro de los ítems se debieron modificar los términos para mantener la equivalencia conceptual con el original. El índice de confiabilidad alfa de Cronbach (0.89), la correlación ítem-escala total (0.41-0.78), el índice test-retest medido mediante el coeficiente de correlación intra-clase (CCI) (0.91) mostraron un nivel satisfactorio. El análisis factorial confirmatorio mostró un RMSEA de 0.054, considerado aceptable; un valor CFI igual a 0.963 , un valor de NNFI 0.925 (levemente inferior al deseado) y un GFI con un valor de 0.991. Se obtuvo una solución de dos factores, por lo cual no se pudo sostener la hipótesis de uni-dimensionalidad de la escala original. Se concluye que la escala traducida y adaptada al español para una muestra de adultos mayores no institucionalizados tiene un ajuste adecuado, con alta consistencia interna y buena confiabilidad test-retest, con iguales valores de constructo que el instrumento original, lo que permitiría su uso en la población adulta mayor.

Palabras clave: adultos mayores, bienestar psicológico, bienestar mental, propiedades psicométricas, análisis factorial confirmatorio
\end{abstract}

\section{TRANSLATION, SPANISH ADAPTATION AND VALIDATION OF THE WARWICK-EDINBURGH WELL-BEING SCALE IN A SAMPLE OF ARGENTINE OLDER ADULTS}

\begin{abstract}
The study assesses psychometric properties and factorial structure of the Warwick-Edinburgh mental well-being scale after forward and backward translations and Spanish adaptation. The scale was validated by applying it to a sample of 910 Argentine elders, with ages ranging from 71 and 96 years (media 81.4 years; SD 10.9 years). The following measures were obtained: response distributions, internal consistency, test-retest reliability, construct validity and exploratory and confirmatory factor analysis. In four of the original items, phrasing was modified to keep conceptual equivalence with the original instrument. Reliability (Cronbach's alpha 0.89), item-total scale (0.41-0.78) and test-retest measured by intra-class correlation coefficient (ICC) (0.91) were deemed appropriate. Confirmatory factorial analysis showed a RMSEA $=0.054$, considered acceptable, a $\mathrm{CFI}=0.963$, a NNFI $=0.925$ (slightly below the accepted value) and a GFI $=0.991$. A two factor solution was obtained so it was not possible to hold the single dimensional hypothesis as in the original scale. The translated and adapted scale shows an appropriate level of adjustment, high internal consistency and good test-retest reliability, with the same construct validity as the original instrument which allows its use among the elderly population.

Key words: Argentine elders, psychological well-being, mental well-being, psychometric properties, confirmatory factor analysis
\end{abstract}

* Zeballos 1625 (2000) Rosario, Santa Fe, República Argentina, teléfono: 54(341) 4215215. danielserrani@argentina.com 


\title{
TRADUÇÃO, ADAPTAÇÃO AO ESPANHOL E VALIDAÇÃO DA ESCALA DE BEM-ESTAR MENTAL DE WARWICK-EDINBURGH EM UMA MOSTRA DE ADULTOS MAIORES ARGENTINOS
}

\begin{abstract}
Resumo
O estudo analisa as propriedades psicométricas e a estrutura fatorial da Escala de Bem-estar Mental de Warwick-Edinburgh depois de realizar a tradução direta e revertida e sua adaptação ao espanhol. Validou-se a escala mediante sua aplicação a uma mostra de 910 adultos maiores argentinos, com idades compreendidas entre 71 e 96 anos (média 81.4 anos; DE 10.9 anos). Obtiveram-se os seguintes indicadores: distribuição de respostas, consistência interna, confiabilidade teste-reteste, validade de constructo, e realizou-se uma análise fatorial exploratória e confirmatória. Em quatro dos itens tiveram que ser modificados os termos para manter a equivalência conceitual com o original. $\mathrm{O}$ índice de confiabilidade alfa de Cronbach (0.89), a correlação item-escala total (0.41-0.78), o índice teste-reteste medido mediante o coeficiente de correlação intra-classe (CCI) (0.91) mostraram um nível satisfatório. A análise fatorial confirmatória mostrou um RMSEA de 0.054, considerado aceitável; um valor CFI igual a 0.963, um valor de NNFI 0.925 (levemente inferior ao desejado) e um GFI com um valor de 0.991. Obteve-se uma solução de dois fatores, pelo qual não de pôde sustentar a hipótese de uni-dimensionalidade da escala original. Concluise que a escala traduzida e adaptada ao espanhol para uma mostra de adultos maiores não institucionalizados tem um ajuste adequado, com alta consistência interna e boa confiabilidade teste-reteste, com iguais valores de constructo que o instrumento original, o que permitiria seu uso na população adulta maior.

Palavras chave: adultos maiores, bem-estar psicológico, bem-estar mental, propriedades psicométricas, análise fatorial confirmatório
\end{abstract}

\section{INTRODUCCIÓN}

A lo largo de la historia se ha considerado a la felicidad como el máximo bien y la motivación más importante para la acción humana, pero el concepto de bienestar psicológico fue ignorado durante mucho tiempo hasta las últimas décadas (Diener, Suh, Lucas, \& Smith, 1999) donde se resumen dos grandes conclusiones: a) la mayor felicidad se asocia con ventajas en salud, educación, sueldos, extroversión, optimismo, religiosidad, autoestima y capacidad de trabajo, independientes del género o rango de inteligencia, y b) hay pocos esfuerzos teóricos para comprender el sentido psicológico de la felicidad.

El concepto del bienestar mental remite a una compleja interacción entre procesos psicológicos, incluyendo afectos negativos y positivos, sentimiento de compromiso y satisfacción duradera con la vida (Diener, Oishi, \& Ryan, 2013). En general, al bienestar mental subjetivo se le encuadra dentro de la Psicología positiva, enfocada en el desarrollo personal, y en los sentimientos subjetivos de satisfacción y logro de la felicidad (Seligman \& Csikszentmihalyi, 2000).

Se ha demostrado la existencia de relaciones entre el bienestar mental y otras variables tales como desarrollo juvenil (Park, 2004), participación social (Nieminen, Martelin, Koskinen, Aro, Alanen, \& Hyyppä, 2010), competencia emocional (Nelis, Kotsou, Quoidbach, Hansenne, Weytens, Dupuis, \& Mikolajczak, 2011), salud física y mental (Zhang, Chen, McCubbin, McCubbin, \& Foley, 2011), planificación del tiempo libre (Adams, Leibbrandt, \& Moon, 2011) y longevidad (Diener \& Chan, 2011), afirmando el valor heurístico del término (Tay \& Diener, 2011; Diener, 2012).

Los estudios de bienestar subjetivo coinciden en que los principales indicadores de optimismo y felicidad se relacionan con una vida con compromisos pero con una sensación de tranquilidad y placidez (Proctor, Maltby, \& Linley, 2011).

Las creencias y emociones positivas, asociadas a la visión hedónica de sí mismo, se relacionan con una mayor amplitud de intereses y recursos de afrontamiento en situaciones cotidianas. Esto desplaza el locus de control de los sucesos hacia el interior del sujeto, favoreciendo una espiral creciente de bienestar mental y resiliencia (Fredrickson \& Joiner, 2002).

Pueden distinguirse dos perspectivas del bienestar mental: la hedónica, enfocada en la experiencia subjetiva de felicidad; y la eudaemónica, dirigida hacia la realización personal (Ryan \& Deci, 2001). En las escalas destinadas a explorar la salud mental se repite esta distinción, de modo que los ítems que exploran los componentes afectivos y los aspectos psicológicos permanecen separados (Waterman, Schwartz, \& Conti, 2008).

Hay investigaciones recientes que no apoyan esta dicotomía, sugiriendo que la salud mental negativa implica limitaciones tanto del bienestar eudaemónico como del hedónico (Henderson \& Knight, 2012; Kashdan, BiswasDiener, \& King, 2008).

Mientras que los jóvenes exhiben como componentes del bienestar la búsqueda de competencia laboral, la práctica 
de actividades placenteras, el logro de relaciones sociales satisfactorias y el autoconocimiento, los adultos mayores enfatizan el afrontamiento positivo de los cambios vitales, la estabilidad emocional y la salud física (Heikkinen \& Kauppinen, 2011).

Si bien se ha intentado establecer una diferenciación entre bienestar subjetivo, como el juicio cognitivo de satisfacción y felicidad, además de las evaluaciones afectivas positivas y negativas (Kim-Prieto, Diener, Tamir, Scollon, \& Diener, 2013) y bienestar psicológico resultante del logro de metas basadas en valores y no solo de actividades placenteras y evitación de situaciones dolorosas (Ryff \& Singer, 2013), en el momento actual hay consenso en que el concepto de bienestar mental o psicológico es multidimensional e incluye al menos seis elementos (van Dierendonck, Díaz, Rodríguez-Carvajal, Blanco, \& Moreno-Jiménez, 2008): a) auto-aceptación, b) propósito en la vida, c) autonomía, d) relaciones positivas con los demás, e) manejo del medio ambiente, y f) crecimiento personal.

Dado que el bienestar subjetivo se asocia con mejores indicadores de salud mental, se han propuesto intervenciones que promuevan mayores índices de salud mental positiva (Clark, Jackson, Carlson, Chou, Cherry, Jordan-Marsh, \& Azen, 2012). No obstante, el campo de la salud mental positiva aún sigue teniendo desventajas debidas a la ausencia de medidas apropiadas basadas en datos poblacionales (McDowell, 2010).

De modo que existe una necesidad de contar con instrumentos confiables para evaluar el bienestar mental en salud pública que no presente problemas de estrechez de rango de medidas (efecto techo) en poblaciones amplias.

Lo anterior ha suscitado un gran interés en la construcción y evaluación de instrumentos que permitan analizar el bienestar mental y su implicación en la psicología positiva y el desarrollo humano. Los instrumentos en este campo se basan en diferentes conceptualizaciones de bienestar. Por ejemplo, la escala de 20 ítems PANAS (Watson, Clark, \& Tellegen, 1988) describe los aspectos afectivos y emocionales del bienestar y comprende dos dimensiones: afecto positivo, y negativo (PANAS-PA y PANAS-NA), reportados como dos constructos distintos e independientes.

El Five-item Satisfaction with Life Scale (SWLS, por sus siglas en inglés) (Diener, Emmons, Larsen, \& Griffin, 1985) mide los aspectos cognitivos del bienestar mental. La Scale of Psychological Well-Being (SPWB, por sus siglas en inglés) de 54 ítems (Ryff \& Keyes, 1995) evalúa el aspecto eudaemónico del bienestar y el funcionamiento psicológico, mientras que las sub-escalas miden autonomía, auto-aceptación, dominio del entorno, propósito en la vida, crecimiento personal y relación positiva con los demás.
La Short Depression-Happiness Scale de cinco ítems (SDHS, por sus siglas en inglés) (Josep, Linley, Harwood, Lewis, \& McCollam, 2004) evalúa el bienestar a lo largo de un continuo entre los dos estados de depresión y felicidad y se usa en el ámbito clínico.

El WHO Well-being Index (WHO-5, por sus siglas en inglés) (Bech, 2004) tiene cinco ítems positivos para medir el bienestar general y valora aspectos de salud física y mental. Existen numerosas escalas pero solamente algunas de ellas han sido traducidas y validadas en español (Cabañero, Richard, Cabrero, Orts, Reig, \& Tosal, 2004; Díaz, Rodríguez, Blanco, Moreno, Gallardo, Valle, \& van Dierendonck, 2006).

La Warwick-Edinburgh Well-Being Scale (WEWBS, por sus siglas en inglés) se desarrolló a partir del Afectómetro 2 (Kammann \& Flett, 1983) compuesto por 20 afirmaciones y 20 adjetivos referidos a la salud mental. A pesar de su validez de constructo y confiabilidad test retest, presentaba limitaciones como consistencia interna elevada, indicativo de redundancia, largo tiempo de administración, y alto sesgo de deseabilidad en las respuestas (Tennant, Joseph, \& Stewart-Brown, 2007a).

Para superar estos inconvenientes se construyó la WEWBS (Tennant, Hiller, Fishwick, Platt, Joseph, Weich, $\&$ Stewart-Brown, 2007b) basada en una concepción del bienestar mental que incluye aspectos hedónicos y eudaemónicos a través de 14 ítems que facilitan su administración en estudios poblacionales. Obtuvo buena validez, confiabilidad test-retest (0.83) y consistencia interna (0.91). El análisis factorial apoyó una solución de un factor.

La escala ha sido validada en grupos e individuos (Maheswaran, Weich, Powell, \& Stewart-Brown, 2012), en poblaciones de diferentes áreas geográficas (Lloyd \& Devine, 2012; Stewart-Brown, Tennant, Tennant, Platt, Parkinson, \& Weich, 2009; Stewart-Brown, 2013), con diferentes profesiones y culturas (Bartram, Sinclair, \& Baldwin, 2013; Taggart, Friede, Weich, Clarke, Johnson, \& Stewart-Brown, 2013), diferentes edades (Clarke, Friede, Putz, Ashdown, Martin, Blake, \& Stewart-Brown, 2011), y capacidades cognitivas (Deary, Watson., Booth, \& Gale, 2013).

Existe una versión en español (López, Gabilondo, Codony, García-Forero, Vilagut, Castellví, \& Alonso, 2012) validada en una muestra de 1900 participantes entre 15 y 70 años (Castellví, Forero, Codony, Vilagut, Brugulat, Medina, \& Alonso, 2013), con alta consistencia interna (alfa de Cronbach $=0.930$ ), buen poder discriminativo y ajuste satisfactorio para soluciones multifactoriales.

Por otra parte, los instrumentos para evaluar el bienestar mental son susceptibles de sesgos debidos a diferencias 
culturales, étnicas o lingüísticas (Ramada, Serra, \& Delclós, 2013) y se ha sugerido que las escalas desarrolladas en países europeos incorporan inadvertidamente una visión individualista de la vida que podría no ajustarse a las concepciones imperantes en las culturas latinas (Christopher, \& Hickinbottom, 2008).

En este último sentido, un instrumento con validez externa de situación o ecológica, aplicable de manera no intrusiva en ámbitos de desempeño social cotidiano representaría una fortaleza metodológica. En efecto, la validez ecológica permitiría obtener conjeturas teniendo en cuenta el contexto donde se producen los fenómenos (Bernal, Bonilla, \& Bellido, 1995).

Teniendo en cuenta las limitaciones ya señaladas con respecto a las versiones anglosajona y española de la WEWBS, se decidió traducir al español y adaptar la Escala de Bienestar Mental Warwick-Edinburgh (EBMWE) para una población de adultos mayores argentinos, para luego verificar sus características psicométricas y establecer su validez externa a través de la convergencia con otros indicadores de bienestar.

\section{MÉTODO}

\section{Diseño}

El diseño del estudio está centrado en la traducción y adaptación de un instrumento de medida: la Escala de Bienestar Mental Warwick-Edinburgh (EBMWE). Este diseño corresponde a la categoría de instrumental (Montero \& León, 2005) porque tiene como objetivo traducir y adaptar una escala y luego realizar un análisis de sus propiedades psicométricas.

\section{Participantes}

La muestra de investigación estuvo formada por 910 adultos mayores argentinos de ambos sexos, con un rango de edades comprendido entre los 71 y los 96 años (media 81.4 años; DE 10.9 años). Respecto al género de los participantes, un $59.1 \%$ correspondió a mujeres y un $40.9 \%$ a hombres. Los participantes fueron seleccionados y entrevistados personalmente durante su asistencia a actividades sociales en cinco centros de jubilados. Luego de una explicación de la naturaleza y el alcance del estudio se obtuvo el consentimiento informado de cada participante. El estudio fue aprobado por el Comité de Ética Local.

\section{Instrumentos de medida}

Escala de bienestar mental de Warwick-Edinburgh (EBMWE). Este instrumento mide los aspectos positivos de salud mental durante las últimas dos semanas. Es una escala ordinal que comprende 14 afirmaciones positi- vas referentes a aspectos hedónicos y eudaemónicos de bienestar mental. Cada ítem se responde sobre la base de una escala Likert de 5 puntos desde "nunca" hasta" todo el tiempo" y el resultado final se obtiene de la suma de todos los ítems (rango 14 a 70 puntos) Los mayores puntajes señalan mayores niveles de bienestar mental.

Escala de bienestar psicológico de Ryff (Díaz et al. 2006). Evalúa el funcionamiento psicológico subjetivo en contraposición a la patología y el malestar. Mide seis dimensiones positivas con seis escalas y 39 ítems con respuesta tipo Likert de 1 (totalmente en desacuerdo) a 6 (totalmente de acuerdo). Tiene buena consistencia interna con un alfa de Cronbach de 0,84 a 0,7.

Escala de salud mental de Goldberg (GHQ-12-General Health Questionnaire) (Rocha, Pérez, Rodríguez-Sanz, Borrell, \& Llandrich, 2011). Esta escala está formada por 12 ítems, siendo 6 de ellos sentencias positivas, y 6 sentencias negativas. Los ítems son contestados a través de una escala tipo Likert de cuatro puntos (0-1-2-3), que puede ser transformada en una puntuación dicotómica (0-0-1-1), llamada puntuación GHQ. Presenta buena fiabilidad (alfa de Cronbach entre 0,82 y 0,86, sensibilidad de 71.2, especificidad de 74.4 y buena validez transcultural. Puntuaciones de 14 y más indican malestar.

Escala de satisfacción con la vida de Diener (Cabañero et al, 2004). Esta es una escala multi-ítem para medir la satisfacción vital. Consta de cinco ítems con alternativa de respuesta múltiple, entre 5 (muy de acuerdo) y 1 (muy en desacuerdo); mostrando buenas propiedades psicométricas (alfa de Cronbach $=0,87$, correlación test-retest $=0,82$ a los dos meses y correlación ítem-test entre 0,57 y 0,75 ).

\section{Análisis estadísticos}

Se midió la consistencia interna con coeficiente alfa de Cronbach. Se efectuó un análisis estadístico de los ítems calculando media, desviación típica, correlación ítem-total y alfa de Cronbach sin el ítem. El poder discriminativo se calculó analizando la diferencia de medias entre los cuartiles superior e inferior del rango de respuestas, con una correlación entre el ítem y la escala no inferior a 0,50 . También se efectuó un test de sedimentación y el número de factores se determinó examinando la última caída sustancial en la magnitud de los valores Eigen (Fabrigar, Wegener, MacCallum, \& Strahan, 1999).

Se llevó a cabo un análisis factorial exploratorio (AFE) con el método de extracción de componentes principales para encontrar los factores de primer orden, con una rotación Oblimin de segundo orden para detectar dimensiones no correlacionadas y variables latentes (Widaman, 1993). Los ítems debían cumplir los siguientes requisitos: a) valor propio mayor o igual a la unidad; b) saturación (carga 
factorial) igual o superior a 0,$40 ;$ c) exclusión de preguntas con cargas similares en distintos factores y agrupables en un ítem dentro del factor con mayor saturación; d) poseer congruencia conceptual entre todas las preguntas incluidas en un factor; e) estar conformado al menos por dos preguntas, a excepción de factores con alto coeficiente de consistencia interna y f) tener confiabilidad superior a 0.50 .

Los análisis de correlación se llevaron a cabo con el coeficiente de Pearson para cada uno de los 14 ítems cuando los datos tenían una distribución normal; de lo contario se calculó el rango de correlación de Spearman. Para evaluar la validez de constructo (consistencia externa) se estableció una correlación de Spearman entre la EBMWE, la escala de bienestar de Ryff, la escala de satisfacción con la vida de Diener y el cuestionario GHE-12.

Para el análisis factorial confirmatorio de la EBMWE (método de estimación: máxima verosimilitud) se empleó el programa AMOS 16.0. Se plantearon cuatro modelos teóricos diferentes. El modelo 1 postula que existe un único factor, llamado bienestar psicológico, en el que saturarían todos los ítems. El modelo 2 defiende una estructura bifactorial donde los ítems referidos al funcionamiento positivo saturarían en un factor y los ítems correspondientes a los afectos positivos en el otro. El modelo 3 propone una estructura de tres factores (emocional, psicológico y social) (Keyes, Ryff, \& Shmotkin, 2002). El modelo 4 postula una estructura teórica de cinco factores (emociones positivas, compromiso, relaciones, significado de la vida, autonomía) según el modelo PERMA (Seligman, 2011). Finalmente, el modelo 5 propone una estructura de seis dimensiones más un factor anidado de segundo orden denominado bienestar social, combinando el bienestar hedónico/emocional (felicidad o satisfacción con la vida) con las 6 dimensiones eudaemónicas del bienestar psicológico de Ryff (van Dierendonck, 2004). La significación estadística se fijó en $\mathrm{p}=0.01$ (dos-colas). Los análisis estadísticos se llevaron a cabo con el paquete estadístico SPSS versión 12.

Cálculo del tamaño muestral. El tamaño muestral se calculó sobre la base de un coeficiente de correlación de Pearson de dos lados estimado de 0.5 , con un error tipo I de 0.001 , un poder de $99 \%$ y un intervalo de confianza de $95 \%$ para el coeficiente de correlación no mayor de 0.5 , lo cual arrojó un número de 103 personas como suficientes para el análisis factorial (Machin, Campbell, Tan, \& Tan, 2011) teniendo en cuenta los informes previos sobre la uni-dimensionalidad de la escala y la presupuesta alta comunalidad. La fórmula utilizada para el cálculo fue:

$$
z=\frac{1}{2} \operatorname{In} \frac{1+r}{1-r} n=3+\frac{4 z_{\alpha} z_{\beta}}{\operatorname{In} \frac{1+r}{1-r} \times \frac{1-r_{0}{ }^{2}}{1+r_{0}}}
$$

\section{Procedimiento}

Para la adaptación del instrumento original se cumplieron los siguientes pasos:

1. Traducción al español por dos traductores independientes (Trust, 1997).

2. Re-traducción por un nuevo traductor bilingüe (Maneersriwongul et al. 2004).

3. Desarrollo de validez aparente y de contenido (Escobar \& Cuervo, 2008) por tres revisores, siguiendo las convenciones habituales (Lawshe, 1975), cumpliendo los criterios de Flaherty (Flaherty, Gaviria, Pathak, Mitchell, Wintrob, Richman, \& Birz, 1988) para asegurar la equivalencia de contenido, semántica, técnica, de criterio y conceptual con el original.

4. Modificación de solo cuatro ítems en la traducción de acuerdo con el criterio de los expertos (véase apéndice 1).

El Coeficiente de validez de contenido fue de 0.77. El cálculo del CVC se basa en el promedio de los puntajes de la escala sobre el número de evaluadores expertos. Polit y colaboradores (Polit, Beck, \& Owen, 2007) desarrollaron una fórmula que integra el valor del CVC junto a un coeficiente de Kappa modificado para prevenir una concordancia entre evaluadores por azar. Los criterios Kappa fueron: aceptable $0.40-0.59$; bueno $0.60-0.73$; y excelente $\geq 0.74$. Los ítems con valores de CVC por debajo de 0.59 fueron considerados potencialmente problemáticos requiriendo una revisión adicional y eventualmente una corrección (Cicchetti \& Sparrow, 1981).

Esta primera versión (versión 1.0) se administró a una muestra piloto de 25 adultos mayores para lograr la adaptación del instrumento, quienes respondieron sobre la claridad y comprensión de la escala; posterior a ello se obtuvo la versión definitiva (versión 1.1). (Véase apéndice 2).

Con esta última se realizaron las entrevistas durante los meses de marzo a noviembre de 2013. Se obtuvieron los datos sociodemográficos de cada participante, luego de lo cual se explicó la naturaleza del cuestionario de manera personal.

Después de completar la EBMWE se administraron los restantes instrumentos para la validación convergente. Durante la aplicación de la EBMWE se aclararon las dudas de los participantes respecto a los ítems, el significado de algunos conceptos y las instrucciones de respuesta. La duración promedio de la aplicación fue de 20 minutos.

Los datos obtenidos fueron codificados y guardados en una planilla Excel y exportados posteriormente a las plataformas SPSS y AMOS. 


\section{RESULTADOS}

La muestra estuvo constituida por adultos mayores con una media de edad aproximada de 80 años y con una mayoría de mujeres, lo que refleja la composición demográfica habitual en esa franja etaria. No hubo, sin embargo, diferencias significativas en las variables estudiadas entre ambos géneros. Las características sociodemográficas detalladas de la muestra se observan en la tabla 1.

En la tabla 2 se presentan los puntajes descriptivos para cada ítem de la Escala de Bienestar Mental para verificar su distribución en la muestra utilizada, así como la correlación ítem-total

Como se puede apreciar, un número importante de ítems presenta medias superiores a 4.0, aunque en general la desviación típica asegura una capacidad de discriminación dentro de los rangos aceptables. El coeficiente de correlación de rangos de Spearman para cada ítem con el resto de la escala fue de 0.51 para los ítems 1 y 10 ("me he sentido optimista con relación al futuro" $y$ "me he sentido con confianza en mi mismo") a 0.78 para el ítem 13 ("he estado interesado por cosas nuevas"), ubicándose la respuesta media por debajo del punto medio de la escala en el ítem 13. Los valores de las desviaciones típicas se situaron por encima de 1 (entre 1,02 en ítem 12 y 1,64 en ítem 2). La correlación entre ítem y la puntuación total de la escala no arrojó ningún ítem por debajo del valor deseable de 0,50. La diferencia entre los cuartiles superior e inferior de respuestas a ítems fue significativa $\left(\mathrm{F}_{(902)}=12,24 ; \mathrm{p}<0.01\right)$ indicando buen poder discriminativo de los ítems. Además, fue homogénea la distribución de frecuencias para las opciones de respuesta a los ítems de la escala, que coincidieron en menos del
$75 \%$ de los casos. La distribución de los puntajes totales resultó normal y homocedástica con promedio de sesgo $=$ -0.05 y de curtosis $=0.01$, por lo que no se realizó ningún procedimiento de transformación de los ítems. En la figura 1 se presenta la distribución de respuestas para la EBMWE, con una distribución normal (media 49.09 IC 95\% 47.09 a 50.15 ) y una mediana de 50 . Estos valores fueron similares a la mediana de 51 encontrada en la muestra poblacional de Escocia (Tennant et al., 2007b) y a la mediana de 51 encontrada en la muestra poblacional de Irlanda del Norte (Lloyd \& Devine, 2012).

El análisis de consistencia interna se llevó a cabo mediante el coeficiente alfa de Cronbach considerado aceptable si tiene un valor $\geq 8$ (George \& Mallery, 2011). El valor alcanzado por la escala fue de 0.89 (IC 95\% 0.85-0.94) indicando un alto nivel de consistencia interna, coincidente con los hallazgos reportados en los trabajos originales de 0.91 (Tennant et al, 2007b) y 0.93 (Lloyd \& Devine, 2012)

La confiabilidad Test-retest se evaluó dentro de un subgrupo de 23 participantes valorados por el mismo investigador con un intervalo promedio de 90 días $(\mathrm{DE}=47)$. El coeficiente de correlación intra-clase fue de 0.91 $(p=0.001)$ sugiriendo buena estabilidad a lo largo del tiempo.

A fin de comprobar la dimensionalidad de la EBMWE, se procedió a realizar en primer lugar un Análisis Factorial Exploratorio (AFE) con el método de extracción de componentes principales con rotación oblicua Oblimin. El análisis de la idoneidad de los datos mediante adecuación muestral de Kaiser-Meyer-Olkin $(\mathrm{KMO}=0.890)$ y una prueba de esfericidad de Barlett significativa $\left(\chi^{2}{ }_{(976)}=5478.92\right.$; $\mathrm{p}<0.001)$ indicaron la viabilidad de los criterios para ser sometidos al análisis factorial, de acuerdo con el criterio

Tabla 1.

Datos sociodemográficos de la muestra

\begin{tabular}{|c|c|c|c|}
\hline \multirow[b]{2}{*}{ Variable } & \multirow{2}{*}{$\begin{array}{l}\text { Hombres }(\mathrm{n}=372) \\
\text { Media }(\mathrm{DE})\end{array}$} & \multicolumn{2}{|c|}{ Mujeres $(n=538)$} \\
\hline & & Media (DE) & $\mathrm{F}(p<.01)$ \\
\hline Edad (años) & $80.7(12.4)$ & $82.1(13.1)$ & .34 \\
\hline Educación (años) & $10.6(4.5)$ & $11.1(3.8)$ & .21 \\
\hline \multicolumn{4}{|l|}{ Estado civil [n (\%)] } \\
\hline Soltero & $24(6.5)$ & $28(5)$ & .04 \\
\hline Casado & $305(82)$ & $468(87)$ & .16 \\
\hline Viudo & $43(11.5)$ & $42(8)$ & .09 \\
\hline
\end{tabular}

Nota. Se consideran como significativos valores $<$ de $.01 ; \mathrm{F}=$ valor de probabilidad; $\mathrm{n}=$ número; $\%=$ valor porcentual; $\mathrm{DE}$ $=$ desviación estándar. 
Tabla 2.

Descriptivos de los ítems de la EBMWE

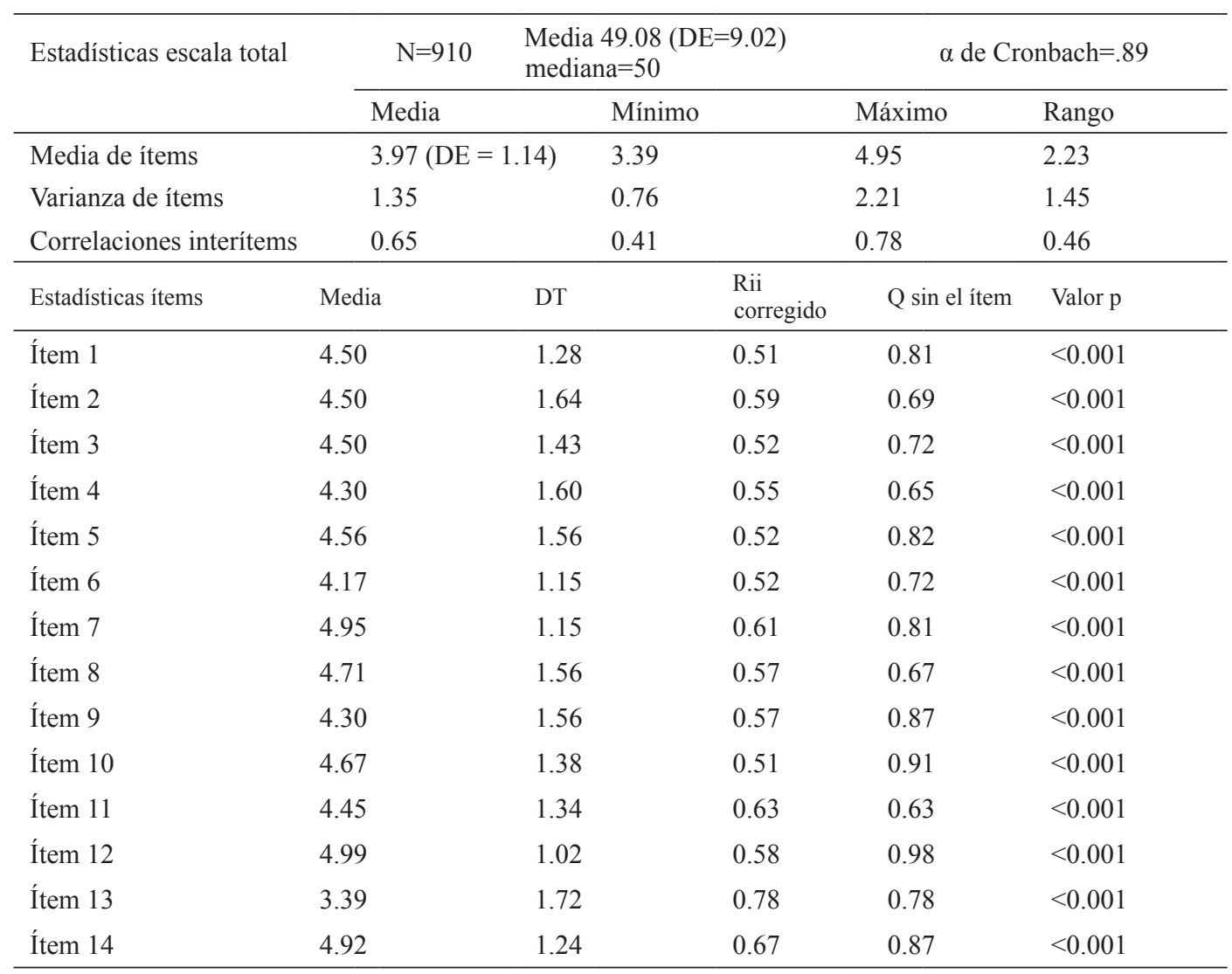

Nota. DT = desviación típica; $\mathrm{N}$ = número total; Rii corregido = relación inter ítem corregido; $\mathrm{Q}$ sin el ítem = correlación de la escala sin el ítem señalado; Valor $\mathrm{p}=$ valor de probabilidad .

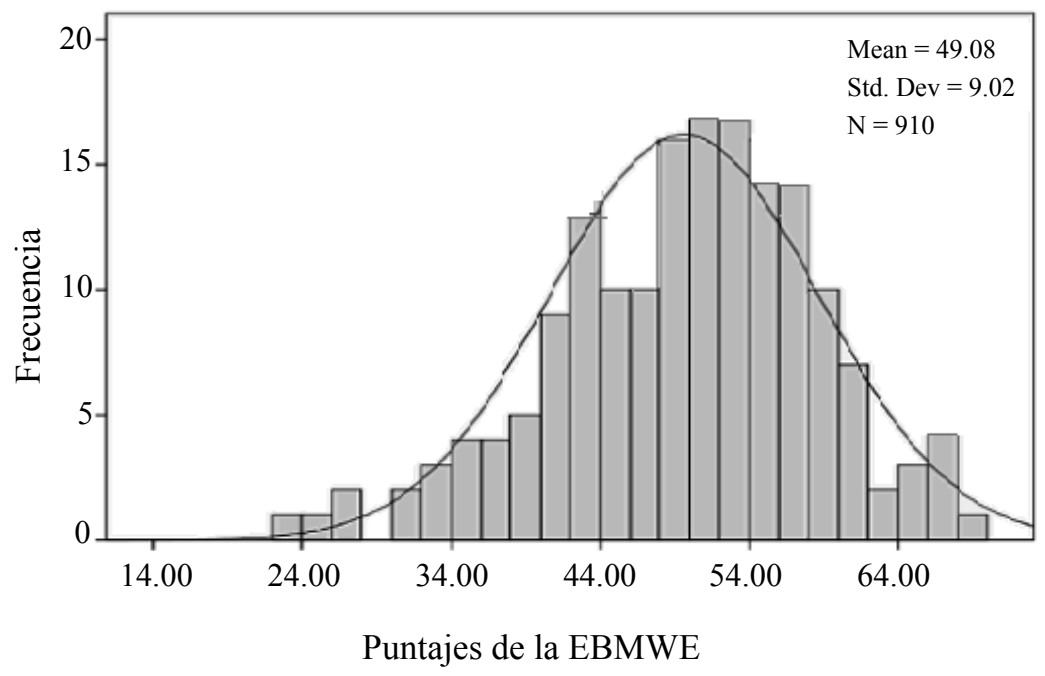

Figura 1. Distribución de puntajes de la EBME en la muestra argentina. Mean = media; Std. Dev $=$ desviación estándar; $\mathrm{N}=$ número total de participantes. 
de Kaiser (Kaiser, 1974) que recomienda valores $\geq 0.5$. Se incluyeron los ítems con saturación $>0.50$, comunalidad $>0.5$ o alto coeficiente de correlación alfa.

Se buscó maximizar la distancia entre factores, que debían tener un auto-valor mayor de 1 , siempre que la varianza total explicada superara el 50\%. Debido al consenso obtenido en estudios anteriores con el modelo de un factor, se exploró este modelo unidimensional. Sin embargo el AFE reveló una estructura bi-factorial que se corresponde con los hallazgos de oros investigadores (Huppert \& So, 2013) y que explicaba el $51 \%$ de la varianza, con cada ítem saturando no menos de 0.5 en el correspondiente factor.

El primer factor comprende optimismo, felicidad, autoestima, auto-confianza, resiliencia y se denominó emociones positivas correspondientes al componente hedónico, en tanto el segundo factor incluyó compromiso, competencia, relaciones y significado personal y se identificó como funcionamiento positivo correspondiente al componente eudaemónico del constructo bienestar mental (véase Tabla 3).

Además del procedimiento de rotación matricial para tratar de identificar los factores de la escala, se realizó un gráfico de sedimentación (screen plot) donde se consideran para la extracción el número de factores presentes hasta el punto en que comienzan a decaer los valores propios. En la muestra del presente trabajo, la mejor solución incluyó dos factores principales. El gráfico mencionado se ofrece en la figura 2.

Tabla 3.

Matriz de componentes rotados de la EQCE oblicua

\begin{tabular}{lll}
\hline \multicolumn{1}{c}{ Ítems } & \multicolumn{2}{c}{ Factor } \\
\cline { 2 - 3 } & $1(51 \%)^{*}$ & $2(29 \%)^{*}$ \\
\hline 1. Me he sentido optimista respecto al futuro & & .32 \\
13. Me he sentido alegre & .67 & .25 \\
3. Me he sentido aliviado & .74 & .35 \\
10. Me he sentido con confianza en mí mismo & .85 & .27 \\
12. Me he sentido querido & .64 & .31 \\
8. Me he sentido bien conmigo mismo & .76 & .43 \\
2. Me he sentido útil & .62 & .31 \\
5. He tenido suficiente energía de reserva & .74 & .32 \\
4. Me he sentido interesado por las demás personas & .76 & .65 \\
6. He resuelto bien los problemas & .32 & .75 \\
11. He sido capaz de tomar mis propias decisiones & .39 & .71 \\
7. He estado pensando con claridad & .12 & .68 \\
14. He estado interesado en cosas nuevas & -.13 & .71 \\
9. Me he sentido cercano a las demás personas & .41 & .69 \\
\hline
\end{tabular}

Nota. $* \%$ de la varianza explicada. 


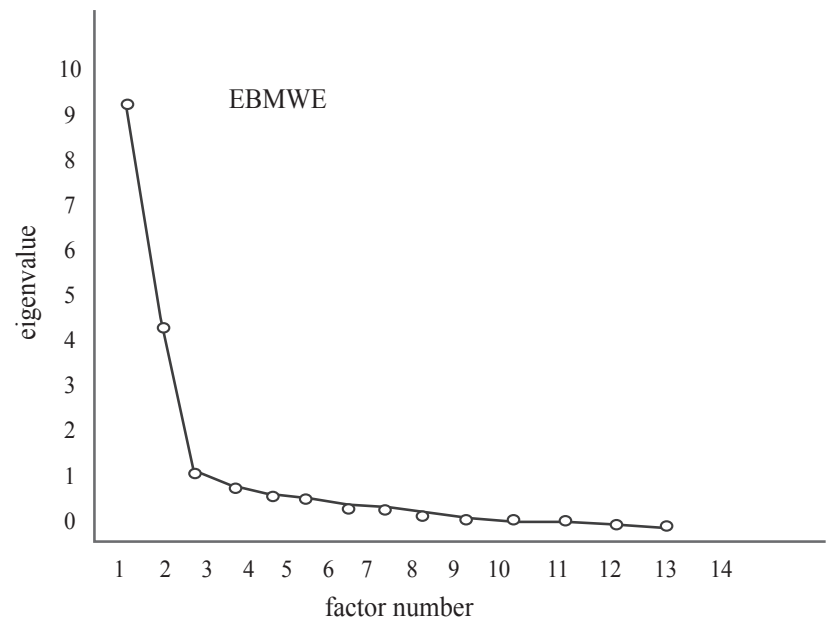

Figura 2. Test de sedimentación para el análisis factorial de la EBMWE.

Por otra parte, el análisis de componentes principales permitió obtener otra serie de valores que confirmaron la existencia de dos factores a partir de los resultados de los auto-valores, los porcentuales de varianza y acumulados. Los dos componentes principales extraídos permitieron explicar el 51 y $29 \%$ de la varianza total, con un total acumulado para los dos factores de casi el 80\%. Los resultados detallados se ofrecen en la tabla 4.

Para establecer el mejor modelo de ajuste, la EBMWE se evaluó con análisis factorial confirmatorio, que permite establecer la bondad de ajuste de diferentes modelos factoriales mediante la aplicación de un modelo de ecuación estructural (MEE). Se evaluaron varios modelos: a) modelo de un factor, b) de dos factores, c) de tres factores y d) de cinco factores. Dado que la bondad de ajuste de Chi cuadrado es muy sensible al tamaño muestral se determinó el mejor ajuste usando índices estandarizados, como el Î́ndice de ajuste comparativo (CFI), Índice de Bondad de ajuste (GFI), Índice de ajuste no normatizado (NNFI) y la raíz de la media del error cuadrado de aproximación (RMSEA). En general, los valores de CFI, GFI y NNFI $\geq .90$ (Hu \& Bentler, 1999), y RMSEA $\leq .06$ indican un buen ajuste empírico (Tabla 5).
Dado que los resultados fueron confirmatorios para un mejor ajuste de modelo bi-factorial, a continuación se llevó a cabo un análisis de sendero de acuerdo con un modelo de ecuación estructural, que permitió determinar los índices de ajuste de cada ítem con los factores, y de cada uno de éstos con la escala total, tal como se ve en la figura 3.

Para la validación externa convergente se examinaron las correlaciones parciales entre los puntajes de la EBMWE y sus dominios con las escalas de Satisfacción con la Vida, la escala de Bienestar Psicológico de Ryff y la GHQ12. El resultado final se muestra en la tabla 6, donde se observa una correlación positiva entre emociones positivas y auto-aceptación junto con la ESV. Por otra parte, hubo correlación directa también entre funcionamiento positivo y relaciones positivas, autonomía y dominio del entorno, ESV y GHQ-12.

\section{DISCUSIÓN}

La adaptación al español de la EBMWE en una población argentina de adultos mayores ofrece una buena validez externa, ya que permite evaluar la mayoría de los conceptos asociados con la salud mental positiva, incluyendo, tanto los aspectos hedónicos, como los eudaemónicos, afectos positivos, relaciones personales satisfactorias y un nivel de funcionamiento general positivo.

En la presente muestra no se observaron efectos de saturación; es decir, el instrumento permitió documentar los cambios en el bienestar mental de manera ajustada a cada participante, como quedó establecido por la amplia diferencia entre los cuartiles superior e inferior de respuestas a los ítems. Lo anterior permite suponer que esta escala resulta adecuada en estudios de intervención donde se requiere detectar los mínimos cambios en bienestar mental como respuesta al tratamiento.

Con respecto a la traducción, sólo cuatro ítems debieron modificarse para mantener su sentido original. El cuestionario final fue similar al usado en otros estudios (López et al., 2012). Esta escala tiene valores adecuados de validez de contenido con alta tasa de respuesta. El análisis factorial

Tabla 4.

Factores de primer orden, auto-valor y porcentaje de varianza explicada

\begin{tabular}{|c|c|c|c|c|c|c|c|c|c|}
\hline \multirow{2}{*}{ Factores } & \multirow{2}{*}{ Media } & \multirow{2}{*}{$\mathrm{DE}$} & \multirow{2}{*}{ Rango } & \multirow{2}{*}{$\alpha$ inicial } & \multirow{2}{*}{ Auto-valor } & \multirow{2}{*}{$\%$ varianza } & \multirow{2}{*}{$\%$ acumulado } & \multicolumn{2}{|c|}{ suma saturaciones ${ }^{2}$} \\
\hline & & & & & & & & \%varianza & \%acumulado \\
\hline 1 & 4.43 & 1.21 & $4.11-4.98$ & .845 & 5.11 & $51 \%$ & $51 \%$ & $51 \%$ & $51 \%$ \\
\hline 2 & 4.15 & 1.03 & $4.02-4.73$ & .841 & 3.10 & $29 \%$ & $80 \%$ & $29.32 \%$ & $80.32 \%$ \\
\hline
\end{tabular}

Nota . Suma saturaciones 2 = suma de saturaciones cuadráticas de la rotación; DE = desviación estándar; $\alpha$ inicial $=$ alfa inicial. 
Tabla 5.

Resultados del Análisis Factorial Confirmatorio

\begin{tabular}{lccccccc}
\hline Modelo & $D f$ & $\mathrm{Chi}^{2}$ & GFI & CFI & NNFI & RMSEA & $N^{\circ}$ ítems \\
1) 1 factor & 904 & 290.5 & .732 & .590 & .601 & 0.093 & 14 \\
2) 2 factores & 904 & 156.2 & $.991^{*}$ & $.963^{*}$ & .925 & $0.054^{*}$ & 14 \\
3) 3 factores & 904 & 399.9 & .643 & .793 & .633 & 0.079 & 14 \\
5) 5 factores & 904 & 275.99 & .771 & .822 & .810 & .0064 & 14 \\
\hline
\end{tabular}

Nota ${ }^{*} \mathrm{p}<0.001 ; \mathrm{Df}=$ grados de libertad; $\mathrm{Chi} 2=$ chi cuadrado; $\mathrm{GFI}=$ índice de bondad de ajuste; $\mathrm{CFI}=$ índice de ajuste comparativo; NNFI = índice de ajuste no normatizado; RMSEA = raíz de la media del error cuadrado de aproximación.

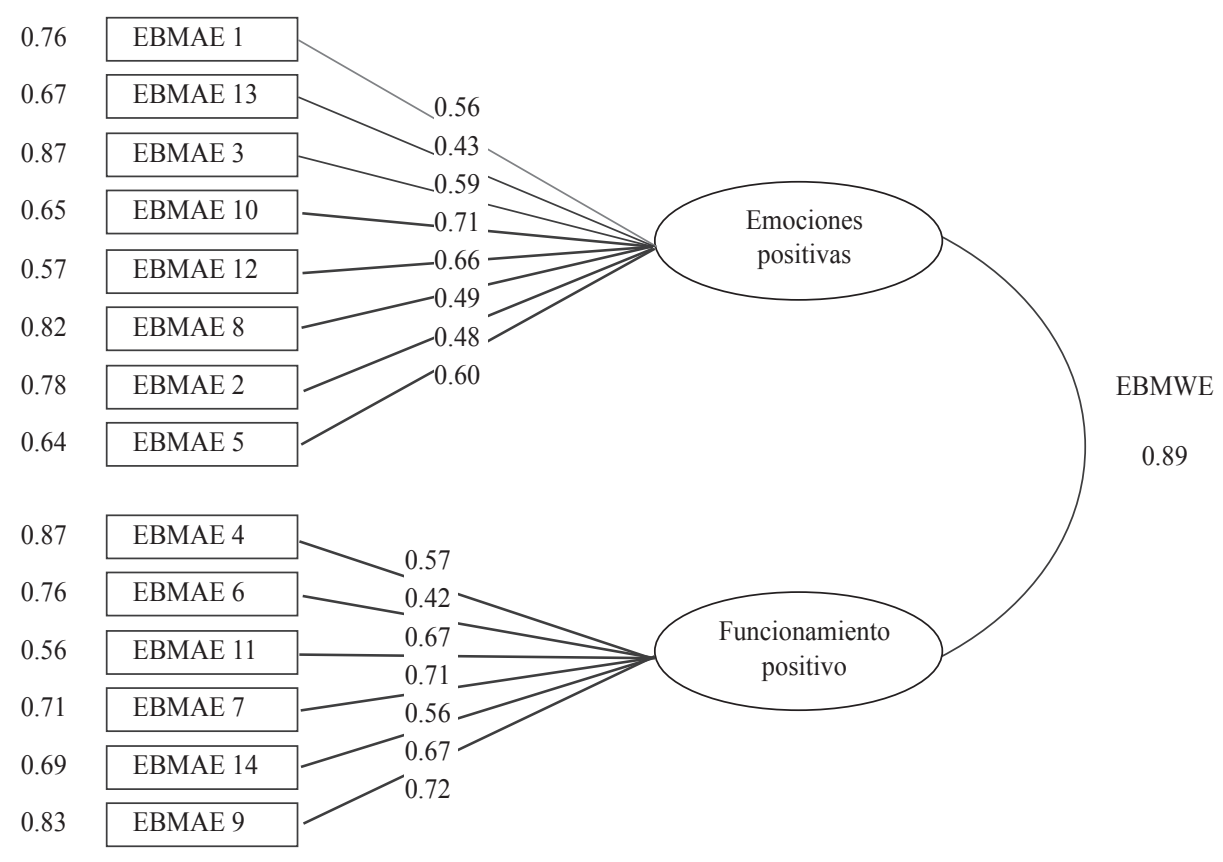

Figura 3. Modelo de Ecuación Estructural para la EBMWE.

confirmatorio permitió obtener como la mejor solución una estructura de dos factores, sugiriendo que este instrumento permite diferenciar entre los componentes psicológicos del bienestar mental y los derivados de las relaciones personales.

Este punto marca una diferencia con las conclusiones de la escala original, donde se apoya una solución unidimensional. El concepto de bienestar mental incluye la respuesta emocional de los sujetos, junto con la valoración global acerca de la satisfacción y conformidad con la vida (Diener, 1984), pero al intentar explicar los procesos que promueven el bienestar mental, la mayoría de los autores incluyen aspectos individuales y situacionales junto con las relaciones sociales (Demir \& Özdemir, 2010). Estas últimas no se limitan a las relaciones íntimas, sino también a las comunitarias (Gracia, \& Herrero, 2006). Es posible que la diferencia factorial encontrada entre el instrumento original y el actualmente validado se debiera a las variaciones existentes en las redes sociales y el contexto socio-político entre adultos mayores europeos y latinos.

Dado que los argentinos mayores tienen altos índices de co-residencia con familiares, la dimensión emocional positiva del bienestar está relacionada con la preserva- 
Tabla 6.

Correlaciones parciales (Rho) de Spearman entre EBMWE y demás instrumentos

\begin{tabular}{lcl}
\hline & \multicolumn{2}{c}{ EBMWE } \\
\cline { 2 - 3 } Escala Bienestar de Ryff & Funcionamiento positivo & Emociones positivas \\
Auto aceptación & $0.24(\mathrm{p}=0.21)$ & $0.51(\mathrm{p}=0.02)$ \\
Relaciones Positivas & $0.67(\mathrm{p}=.0 .01)$ & $0.33(\mathrm{p}=0.43)$ \\
Autonomía & $0.51(\mathrm{p}=0.05)$ & $0.42(\mathrm{p}=0.12)$ \\
Dominio del Entorno & $0.55(\mathrm{p}=0.08)$ & $0.24(\mathrm{p}=0.49)$ \\
Propósito en la Vida & $0.21(\mathrm{p}=0.17)$ & $0.37(\mathrm{p}=0.38)$ \\
Crecimiento Personal & $0.25(\mathrm{p}=0.73)$ & $0.43(\mathrm{p}=0.19)$ \\
ESV & $0.47(\mathrm{p}=0.03)$ & $0.55(\mathrm{p}=0.04)$ \\
GHQ-12 & $0.62(\mathrm{p}=0.04)$ & $0.34(\mathrm{p}=0.12)$ \\
\hline
\end{tabular}

Nota. ESV: escala de satisfacción con la vida; GHQ-12: escala de salud general de 12 ítems.

ción de la estabilidad y cohesión familiar, en tanto que el desempeño positivo cumple un papel relevante en mantener la independencia y autonomía, contribuyendo a diferenciar ambos conceptos.

La consistencia interna de la escala fue alta y no se encontró redundancia entre los ítems. La correlación entre los resultados generales y parciales de la escala con otros instrumentos de medida de bienestar psicológico mostró adecuados índices de concordancia, excepto para el subíndice de emociones positivas de la EBMWE y el GHQ12. Este último detecta dos tipos de trastornos: disfunción social y fenómenos estresantes, los cuales se manifiestan en el adulto mayor en un plano somático con más frecuencia que en el emocional, limitando las relaciones personales.

Es por esto que posiblemente se encuentre una correlación más robusta entre GHQ-12 y las medidas de funcionamiento positivo. Por lo demás, la dirección de las asociaciones estuvo en la dirección esperada, es decir positiva.

Como toda investigación, el presente estudio no está exento de limitaciones. Por un lado, sólo se utiliza una muestra de adultos mayores de una ciudad, por lo que representa una observación parcial de lo que sucede en el conjunto de sujetos y, por lo tanto, restringe la generalización de los resultados a otros ámbitos.

Una segunda limitación se relaciona con la utilización de escalas de auto-informe, que generalmente derivan en información sesgada al tratarse de una mirada personal y subjetiva en que la mayoría de las personas tiende a evaluarse positivamente.
Una tercera limitación está representada por la falta de evaluación del desempeño de la escala para detectar cambios en la salud mental después de la ocurrencia de eventos significativos vitales, o posterior a alguna intervención, lo cual representa un punto importante que debe investigarse en el futuro.

Si bien el objetivo del estudio fue adaptar la EBMWE a población argentina, y evaluar sus características psicométricas y de desempeño, este es un punto inicial, con la finalidad de disponer de instrumentos estandarizados a la realidad local con normas o baremos propios.

Sería deseable, en ese sentido, incrementar la muestra del estudio o realizar otras investigaciones con otras muestras diferentes o complementarias, permitiendo que la realidad investigada sea más heterogénea, a fin de tener una visión más amplia del comportamiento de la escala en otros contextos del país y la región, además de abarcar a otros grupos etarios.

El diseño exhibe fortalezas metodológicas y puede considerarse robusto en su validez externa, al haber sido aplicado en ambientes cotidianos. En ese sentido, las conclusiones obtenidas fueron válidas ecológicamente dado que las conjeturas tuvieron en cuenta el contexto de producción de los fenómenos

La EBMWE muestra niveles de consistencia interna y confiabilidad adecuados, además de ser relativamente breve en su aplicación, comprensible y relevante para grupos poblacionales amplios. 
Puede aplicarse para satisfacer las necesidades de investigaciones en el campo de la salud mental, tanto teóricas como de intervención y promoción, pero se requiere más investigación para asegurar que la escala es suficientemente sensible para detectar cambios.

La posibilidad de desempeño con fuertes propiedades psicométricas y ausencia de efecto de saturación sugieren que es adecuada para medir el bienestar mental a niveles poblacionales amplios en muestras locales.

\section{REFERENCIAS}

Adams, K. B., Leibbrandt, S., \& Moon, H. (2011). A Critical Review of the Literature on Social and Leisure Activity and Well-being in Later Life. Ageing and Society, 31(4), 683

Bartram, D. J., Sinclair, J. M., \& Baldwin, D. S. (2013). Further validation of the Warwick-Edinburgh Mental Well-being Scale (WEMWBS) in the UK veterinary profession: Rasch analysis. Quality of Life Research, 1-13.

Bech, P. (2004). Measuring the Dimensions of Psychological General Well-Being by the WHO-5. Quality of Life Newsletter, 32, 15-16.

Bernal, G., Bonilla, J., \& Bellido, C. (1995). Ecological Validity and Cultural Sensitivity for Outcome Research: Issues for the Cultural Adaptation and Development of Psychosocial Treatments with Hispanics. Journal of Abnormal Child Psychology, 23(1), 67-82.

Cabañero, M. J., Richard, M., Cabrero, J., Orts, I., Reig, A., y Tosal, B. (2004). Fiabilidad y validez de la Escala de Satisfacción con la Vida de Diener en una muestra de mujeres embarazadas y puérperas. Psicothema, 16, 448-455.

Castellví, P., Forero, C. G., Codony, M., Vilagut, G., Brugulat, P., Medina, A., \& Alonso, J. (2013). The Spanish Version of the Warwick-Edinburgh Mental Well-Being Scale (WEMWBS) is Valid for Use in the General Population. Quality of Life Research, 1-12.

Christopher, J. C., Hickinbottom, S. (2008). Positive Psychology, Ethnocentrism, and the Disguised Ideology of Individualism. Theory \& Psychology, 18(5), 563-589.

Cicchetti, D.V., Sparrow, S. (1981). Developing Criteria for Establishing Interrater Reliability of Specific Items: Application To Assessment of Adaptive Behavior. American Journal of Mental Deficiency, 86, 127-137.

Clark, F., Jackson, J., Carlson, M., Chou, C. P., Cherry, B. J., Jordan-Marsh, M., \& Azen, S. P. (2012). Effectiveness of a Lifestyle Intervention in Promoting the Well-Being of Independently Living Older People: Results of the Well Elderly 2 Randomised Controlled Trial. Journal of Epidemiology and Community Health, 66(9), 782-790.

Clarke, A., Friede, T., Putz, R., Ashdown, J., Martin, S., Blake, A., \& Stewart-Brown, S. (2011). Warwick-Edinburgh Mental Well-being Scale (WEMWBS): Validated for
Teenage School Students in England and Scotland. A Mixed Methods Assessment. BMC Public Health, 11(1), 487.

Deary, I. J., Watson, R., Booth, T., Gale, C. R. (2013). Does Cognitive Ability Influence Responses to the WarwickEdinburgh Mental Well-Being Scale? Psychological Assessment; 25(2), 313-8.

Demir, M., \& Özdemir, M. (2010). Friendship, Need Satisfaction and Happiness. Journal of Happiness Studies, 11, 243-259.

Díaz, D., Rodríguez, R., Blanco, A., Moreno, B., Gallardo, I., Valle, C. y van Dierendonck, D. (2006). Adaptación española de las Escalas de Bienestar Psicológico de Ryff. Psicothema, 18(3), 572-577.

Diener, E. (1984). Subjective Well-Being. Psychological Bulletin, 95, 542-575.

Diener, E. (2012). New Findings and Future Directions for Subjective Well-Being Research. American Psychologist, 67(8), 590.

Diener, E., \& Chan, M. Y. (2011). Happy People Live Longer: Subjective Well-Being Contributes to Health and Longevity. Applied Psychology: Health and Well-Being, 3(1), 1-43.

Diener, E., Emmons, R. A., Larsen, R. J., Griffin, S. (1985). The Satisfaction with Life Scale. Journal of Personality Assessment, 49(1): 71-75.

Diener, E., Oishi, S., \& Ryan, K. L. (2013). Universals and Cultural Differences in the Causes and Structure of Happiness: A Multilevel Review. In: Mental Well-Being (pp. 153-176). Springer Netherlands.

Diener, E., Suh, E. M., Lucas, R. E., \& Smith, H. L. (1999). Subjective Well-Being: Three Decades of Progress. Psychological Bulletin, 125(2), 276.

Escobar, J. \& Cuervo, Á. (2008). Validez de contenido y juicio de expertos: una aproximación a su utilización. Avances en Medición, 6(1), 29.

Fabrigar, L. R., Wegener, D. T., MacCallum, R. C., Strahan, E. J. (1999). Evaluating the Use of Exploratory Factor Analysis in Psychological Research. Psychological Methods, 4(3), 272-299.

Flaherty, J. A., Gaviria, F. M., Pathak, D., Mitchell, T., Wintrob, R., Richman, J. A., Birz, S. (1988). Developing Instruments for Cross-Cultural Psychiatric Research. Journal of Nervous \& Mental Disease, 176(5), 257-263.

Fredrickson, B. L., \& Joiner, T. (2002). Positive Emotions Trigger Upward Spirals toward Emotional Well-Being. Psychological Science, 13(2), 172-175.

George, D., \& Mallery, P. (2011). IBM SPSS Statistics 21 Step by Step: A Simple Guide and Reference (13th ed.). Pearson Higher Ed: USA.

Gracia, E., \& Herrero, J. (2006). La comunidad como fuente de apoyo social: evaluación e implicaciones en los ámbitos individual y comunitario. Revista Latinoamericana de Psicología, 38, 327-342. 
Heikkinen, R. L., \& Kauppinen, M. (2011). Mental Well-Being: A 16-Year Follow-Up among Older Residents in Jyväskylä. Archives of Gerontology and Geriatrics, 52(1), 33-39.

Henderson, L.W., \& Knight, T. (2012). Integrating the Hedonic and Eudaimonic Perspectives to Morecomprehensively Understand Well-being And Pathways to Well-being. International Journal of Well-being, 2(3), 196-221.

Hu, L. y Bentler, P. M. (1999). Cutoff Criteria for Fit Indexes in Covariance Structure Analysis. Conventional Criteria versus New Alternatives. Structural Equation Modeling, 6, $1-55$.

Huppert, F. A. and So, T. T. (2013). Flourishing Across Europe: Application of a New Conceptual Framework for Defining Well-Being. Social Indicators Research, 110, 837-861.

Joseph, S., Linley, P. A., Harwood, J., Lewis, C. A., McCollam, P. (2004). Rapid Assessment of Well-Being: The Short Depression-Happiness Scale (SDHS). Psychology and Psychotherapy, 77, 463-478.

Kaiser, H. F. (1974). An Index of Factorial Simplicity. Psychometrica, 39, 31-36

Kammann, R., Flett, R. (1983). A Scale to Measure Current Level of General Happiness. Australian Journal of Psychology, 35, 259-265.

Kashdan, T. B., Biswas-Diener, R., \& King, L. A. (2008). Reconsidering Happiness: The Costs of Distinguishing Between Hedonics and Eudaimonia. The Journal of Positive Psychology, 3(4), 219-233.

Keyes, C., Ryff, C., y Shmotkin, D. (2002). Optimizing WellBeing: The Empirical Encounter of Two Traditions. Journal of Personality and Social Psychology, 82, 1007-1022.

Kim-Prieto, C., Diener, E., Tamir, M., Scollon, C., \& Diener, M. (2013). Integrating the Diverse Definitions of Happiness: A Time-Sequential Framework of Subjective Well-Being. In The Exploration of Happiness (pp. 47-75). Springer, Netherlands.

Lawshe, C. H. (1975). A Quantitative Approach to Content Validity. Personnel Psychology Inc, 28, 563-575.

Lloyd, K., \& Devine, P. (2012). Psychometric Properties of the Warwick-Edinburgh Mental Well-Being Scale (WEMWBS) in Northern Ireland. Journal of Mental Health, 21(3), 257-263.

López, M. A., Gabilondo, A., Codony, M., García-Forero, C., Vilagut, G., Castellví, P., \& Alonso, J. (2012). Adaptation into Spanish of the Warwick-Edinburgh Mental Well-being Scale (WEMWBS) and Preliminary Validation in a Student Sample. Quality of Life Research, 1-6.

Machin, D., Campbell, M. J., Tan, S. B., \& Tan, S. H. (2011). Sample Size Tables for Clinical Studies. John Wiley \& Sons: Chichester, U.K.

Maheswaran, H., Weich, S., Powell, J., \& Stewart-Brown, S. (2012). Evaluating the Responsiveness of the Warwick Edinburgh Mental Well-Being Scale (WEMWBS): Group and Individual Level Analysis. Health Quaity of Life Outcomes, 10(1), 156.

Maneersriwongul, W., Dixon, J. K. (2004). Instrument Translation Process: A Methods Review. Journal of Advanced Nursing 48(2), 175-186.

McDowell, I. (2010). Measures of Self-Perceived Well-Being. Journal of Psychosomatic Research, 69(1), 69-79.

Montero, I. y León, O. (2005). Sistema de clasificación del método en los informes de investigación en psicología. International Journal of Clinical and Health Psychology, 5(1), 115-127.

Nelis, D., Kotsou, I., Quoidbach, J., Hansenne, M., Weytens, F., Dupuis, P., \& Mikolajczak, M. (2011). Increasing Emotional Competence Improves Psychological and Physical WellBeing, Social Relationships, and Employability. Emotion, 11(2), 354 .

Nieminen, T., Martelin, T., Koskinen, S., Aro, H., Alanen, E., \& Hyyppä, M. T. (2010). Social Capital as a Determinant of Self-Rated Health and Psychological Well-Being. International Journal of Public Health, 55(6), 531-542.

Park, N. (2004). The Role of Subjective Well-Being in Positive Youth Development. The Annals of the American Academy of Political and Social Science, 591(1), 25-39.

Polit, D.F., Beck, C.T., Owen, S.V., (2007). Is the CVI an Acceptable Indicator of Content Validity? Appraisal and Recommendations. Research in Nursing \& Health 30, 459-467.

Proctor, C., Maltby, J., \& Linley, P. A. (2011). Strengths Use as a Predictor of Well-Being and Health-Related Quality of Life. Journal of Happiness Studies, 12(1), 153-169.

Ramada-Rodilla, J. M., Serra-Pujadas, C., Delclós-Clanchet, G. L. (2013). Adaptación cultural y validación de cuestionarios de salud: revisión y recomendaciones metodológicas. Salud Pública Mex, 55, 57-6.

Rocha, K. B., Pérez, K., Rodríguez-Sanz, M., Borrell, C., \& Llandrich, J. O. (2011). Propiedades psicométricas y valores normativos del General Health Questionnaire (GHQ-12) en población general española. International Journal of Clinical and Health Psychology, 11(1), 125-139.

Ryan, R. M., \& Deci, E. L. (2001). On Happiness and Human Potential: A Review of Research on Hedonic and Eudaimonic Well-Being. Annual Review of Psychology, 52, 141-166.

Ryff, C. D., \& Singer, B. H. (2013). Know Thyself and Become What You Are: A Eudaimonic Approach to Psychological Well-Being. In The Exploration of Happiness (pp. 97-116). Springer Netherlands.

Ryff, C. D., Keyes, C. L. (1995). The Structure of Psychological Well-Being Revisited. Journal of Personality and Social Psychology, 69, 719-727.

Seligman, M. E. (2011). Flourish. North Sydney, N.S.W.: Random House Australia. 
Seligman, M. E., \& Csikszentmihalyi, M. (2000). Positive Psychology: An Introduction. American Psychologist, 55(1), 5.

Stewart-Brown, S. (2013). The Warwick-Edimburgh Mental Well-Being Scale (WEMWBS): Performance in Different Cultural and Geographical Groups. In C.L.M. Keyes (Ed.), Mental Well-Being: International Contributions to the Study of Positive Mental Health. (pp. 133-150). Dordrecht, Netherlands: Springer.

Stewart-Brown, S., Tennant, A., Tennant, R., Platt, S., Parkinson, J., \& Weich, S. (2009). Internal Construct Validity of the Warwick-Edinburgh Mental Well-Being Scale (WEMWBS): A Rasch Analysis Using Data from the Scottish Health Education Population Survey. Health and Quality of Life Outcomes, 7(1), 15-22.

Taggart, F., Friede, T., Weich, S., Clarke, A., Johnson, M., \& Stewart-Brown, S. (2013). Cross Cultural Evaluation of the Warwick-Edinburgh Mental Well-Being Scale (WEMWBS)-A Mixed Methods Study. Health and Quality of Life Outcomes, 11(1), 27.

Tay, L., \& Diener, E. (2011). Needs and Subjective WellBeing around the World. Journal of Personality and Social Psychology, 101(2), 354.

Tennant, R., Joseph, S., Stewart-Brown, S. (2007a). The Affectometer 2: A Measure of Positive Mental Health in UK Populations. Quality of Life Research, 16(4), 687-695.

Tennant, R., Hiller, L., Fishwick, R., Platt, S., Joseph, S., Weich, S., \& Stewart-Brown, S. (2007b). The Warwick-Edinburgh Mental Well-Being Scale (WEMWBS): Development and UK Validation. Health and Quality of Life Outcomes, 5(1), 63.
Trust, M. O. (1997). Trust Introduces New Translation Criteria. Medical Outcomes Trust Bulletin, 5, 3-4.

Van Dierendonck, D. (2004) The Construct Validity of Ryff's Scale of Psychological Well-Being and its Extension with Spiritual Well-Being. Personality and Individual Differences, 36(3), 629-644.

Van Dierendonck, D., Díaz, D., Rodríguez-Carvajal, R., Blanco, A., \& Moreno-Jiménez, B. (2008). Ryff's Six-Factor Model of Psychological Well-Being, A Spanish Exploration. Social Indicators Research, 87(3), 473-479.

Waterman, A. S., Schwartz, S., \& Conti, R. (2008). The Implications of Two Conceptions of Happiness (Hedonic Enjoyment and Eudaimonia) For the Understanding of Intrinsic Motivation. Journal of Happiness Studies, 9(1), 41-79.

Watson, D., Clark, L. A., Tellegen, A. (1988). Development and Validation of Brief Measures of Positive and Negative Affect: the PANAS Scales. Journal of Personality and Social Psychology, 6(54), 1063-70.

Widaman, K. F. (1993). Common Factor Analysis versus Principal Component Analysis: Differential Bias in Representing Model Parameters? Multivariate Behavioral Research, 28(3), 263-311.

Zhang, W., Chen, Q., McCubbin, H., McCubbin, L., \& Foley, S. (2011). Predictors of Mental and Physical Health: Individual and Neighborhood Levels of Education, Social Well-Being, and Ethnicity. Health \& Place, 17(1), 238-247. 


\section{APÉNDICE 1}

Equivalencia de traducciones entre la versión inglesa y la versión argentina

\begin{tabular}{|c|c|}
\hline Versión español & Versión inglés \\
\hline 3. Me he sentido aliviado & I've been feeling relaxed \\
\hline 6. He resuelto bien los problemas & I've been dealing with problems well \\
\hline $\begin{array}{l}\text { 10. Me he sentido con confianza en mí } \\
\text { mismo }\end{array}$ & I've been feeling confident \\
\hline $\begin{array}{l}\text { 11. He sido capaz de tomar mis propias } \\
\text { decisiones con respecto a las cosas que me } \\
\text { suceden }\end{array}$ & $\begin{array}{l}\text { I've been able to make up my own mind } \\
\text { about things }\end{array}$ \\
\hline 12. Me he sentido querido & I've been feeling loved \\
\hline
\end{tabular}

LA ESCALA DE BIENESTAR MENTAL DE WARWICK-EDINBURGH (EBMWE)

A continuación encontrará algunas afirmaciones acerca de ideas y emociones.

Tenga a bien tildar el casillero que mejor describa lo que pensó o sintió durante las últimas 2 semanas

\begin{tabular}{lccccc}
\multicolumn{1}{c}{ AFIRMACIONES } & $\begin{array}{c}\text { En ningún } \\
\text { momento }\end{array}$ & Raramente & $\begin{array}{c}\text { Algunas } \\
\text { veces }\end{array}$ & $\begin{array}{c}\text { Con } \\
\text { frecuencia }\end{array}$ & $\begin{array}{c}\text { Todo el } \\
\text { tiempo }\end{array}$ \\
\hline $\begin{array}{l}\text { Me he sentido optimista con relación al } \\
\text { futuro }\end{array}$ & 1 & 2 & 3 & 4 & 5 \\
Me he sentido útil & 1 & 2 & 3 & 4 & 5 \\
Me he sentido aliviado & 1 & 2 & 3 & 4 & 5 \\
$\begin{array}{l}\text { Me he sentido interesado por las demás } \\
\text { personas }\end{array}$ & 1 & 2 & 3 & 4 & 5 \\
He tenido suficiente energía de reserva & 1 & 2 & 3 & 4 & 5 \\
$\begin{array}{l}\text { He resuelto bien los problemas } \\
\text { He estado pensando con claridad }\end{array}$ & 1 & 2 & 3 & 4 & 5 \\
$\begin{array}{l}\text { Me he sentido bien conmigo mismo } \\
\text { Me he sentido cercano a las demás per- }\end{array}$ & 1 & 2 & 3 & 4 & 5 \\
$\begin{array}{l}\text { sonas } \\
\text { Me he sentido con confianza en mí } \\
\text { mismo }\end{array}$ & 1 & 2 & 3 & 4 & 5 \\
$\begin{array}{l}\text { He sido capaz de tomar mis propias } \\
\text { decisiones con respecto a las cosas que } \\
\text { me suceden }\end{array}$ & 1 & 2 & 3 & 4 & 4 \\
$\begin{array}{l}\text { Me he sentido querido } \\
\text { He estado interesado en cosas nuevas }\end{array}$ & 1 & 2 & 3 & 4 & 5 \\
\hline \begin{tabular}{l} 
Me he sentido alegre \\
\hline
\end{tabular} & 1 & 2 & 3 & 4 & 5 \\
\hline
\end{tabular}

Warwick-Edinburgh Mental Well-Being Scale (WEMWBS)

(C) NHS Health Scotland, University of Warwick and University of Edinburgh, 2006, all rights reserved. 